\title{
Consideration of the Effect of the Nonspherical Distribution of Electrons in Atomic Structures
}

\author{
Mitiyasu Miyasita ${ }^{1, *}$, Katsuhiko Higuchi ${ }^{2}$, Akira Narita $^{3}$ and Masahiko Higuchi ${ }^{1}$ \\ ${ }^{1}$ Graduate School of Science and Engineering, Shinshu University, Matsumoto 390-8621, Japan \\ ${ }^{2}$ Graduate School of Advanced Science of Matter, Hiroshima University, Higashi-Hiroshima 739-8527, Japan \\ ${ }^{3}$ Division of Applied Mathematics, Akita National College of Technology, Akita 011-8511, Japan
}

\begin{abstract}
We review the atomic structures by taking into account the effect of the nonspherical distribution of electrons explicitly. Actual calculations are performed for the neutral first-row atoms by means of the variational method. Compared to the conventional atomic structures, degenerate energy levels are split partially due to the lack of the spherical symmetry. The magnitudes of these splittings are not negligibly small. This means that the nonspherical part of electron distribution seems to be essential not only conceptually but also quantitatively in the study on the single-particle picture of atomic systems. [doi:10.2320/matertrans.MER2008121]
\end{abstract}

(Received April 7, 2008; Accepted May 15, 2008; Published June 25, 2008)

Keywords: nonspherical distribution of electrons, spherical approximation, atomic structure, first-row atoms, single-particle picture

\section{Introduction}

The single-particle wave functions and spectra for atomic systems have been quite useful for understanding the electronic properties of solids, for instance, estimations of the hopping and Coulomb integrals included in the model Hamiltonian, ${ }^{1,2)}$ the LCAO method $^{3)}$ and the $\mathrm{LDA}+U$ method, ${ }^{4,5)}$ etc. However, there exist problems in the conventional single-particle picture of the atomic structures, which have been pointed out by several workers. ${ }^{6-12)}$ In the conventional atomic structure, the eigenstates are specified by the quantum numbers $n, l, m, \sigma$, which are the principal, azimuthal, magnetic and spin quantum numbers, respectively. ${ }^{13)}$ In other words, the calculation of atomic structures has usually been based on the spherical approximation of the electron distribution. However, such a picture is obviously inappropriate for the open shell atoms due to the lack of the spherical symmetry of the electron distribution. As easily understood, orbitals of different $m$ do not have equivalent densities. This is problematic from the viewpoint of the density functional theory (DFT), ${ }^{14,15)}$ because the electron density and external potential have one-to-one correspondence to each other. Of course, in the first place, the states specified by $n, l, m, \sigma$ are not the self-consistent solutions for the single-particle equation, whether it is the Kohn-Sham equation of the DFT, the Hartree-Fock equation or the other equation.

Slater's proposal would be the first one to modify the conventional single-particle picture of atomic systems. ${ }^{6)}$ But, unfortunately, it has not been performed due to the necessity of the heavy calculation tasks. After Slater's proposal, there have been two kinds of approaches to this problem. One is the variational method where the single-particle wave function is expanded by using appropriately chosen basis functions. ${ }^{7,8)}$ Another is the density functional scheme containing the effect of the orbital current density explicitly. ${ }^{9-12,16-19)}$ In this paper, we adopt the former approach. As the basis functions, eigenfunctions for the spherical part of the single-particle potential are used and updated for each iteration of the selfconsistent calculations. They are apparently different from those of the previous works. ${ }^{7,8)}$

The aim of the present work is to investigate to what extent the single-particle picture of atomic systems needs to be modified. In the following sections we will show the necessity of modifying the single-particle picture of atomic systems not only conceptually but also substantially.

Organization of this paper is as follows. In Sec. 2, we present a scheme for calculating the atomic structures with taking into account the effect of the nonspherical distribution of electrons. The calculation results for the neutral first-row atoms are shown in Sec. 3. The effect of the nonspherical electron density on the atomic structures is also discussed. Some concluding remarks are given in Sec. 4.

\section{A Variational Scheme for Calculating Atomic Struc- tures beyond the Spherical Approximation}

In this paper, neglecting the relativistic effects, we adopt the Hartree-Fock-Slater (HFS) equation ${ }^{20-23)}$ as an effective single-particle equation. First, we expand the effective potential with the spherical harmonics:

$$
V_{\sigma}(\mathbf{r})=\sum_{l m}\left\{v_{l m}^{\sigma}(r) Y_{l m}(\hat{\mathbf{r}})+v_{l m}^{\sigma}(r)^{*} Y_{l m}(\hat{\mathbf{r}})^{*}\right\},
$$

where $v_{l m}^{\sigma}(r)$ are the radial components and their explicit forms are given in Appendix, and where $\hat{\mathbf{r}}$ stands for the angular variables. In a similar way to eq. (1), we shall expand the solution of HFS equation with the set of known basis functions. As the known functions, we here use the eigenfunctions for the Hamiltonian $\hat{H}_{0}^{\sigma}$ that has the spherically-averaged potential calculated from eq. (1). These radial wave functions are calculated easily by means of the numerical methods such as the Herman-Skillman method. ${ }^{24)}$ Thus, the solution of HFS equation is written as

$$
\psi_{i \sigma}(\mathbf{r})=\frac{1}{r} \sum_{n l m} C_{n l m}^{i \sigma} p_{n l}^{\sigma}(r) Y_{l m}(\hat{\mathbf{r}}) .
$$


Substituting eqs. (1) and (2) into the HFS equation, and writing distinctly the spherical and nonspherical parts of the effective potential, we have

$$
\begin{aligned}
\hat{H}_{0}^{\sigma} \sum_{N} & \sum_{L} C_{N L}^{i \sigma} p_{N}^{\sigma}(r) Y_{L}(\hat{\mathbf{r}}) \\
& +\sum_{L^{\prime} \neq(00)}\left\{v_{L^{\prime}}^{\sigma}(r) Y_{L^{\prime}}(\hat{\mathbf{r}})+v_{L^{\prime}}^{\sigma}(r)^{*} Y_{L^{\prime}}(\hat{\mathbf{r}})^{*}\right\} \sum_{N} \sum_{L} C_{N L}^{i \sigma} p_{N}^{\sigma}(r) Y_{L}(\hat{\mathbf{r}}) \\
= & \varepsilon_{i \sigma} \sum_{N} \sum_{L} C_{N L}^{i \sigma} p_{N}^{\sigma}(r) Y_{L}(\hat{\mathbf{r}}),
\end{aligned}
$$

where $n l$ is abbreviated as " $N$ " and $l m$ as " $L$ " for ease of seeing. It can be easily shown that eq. (3) is reduced to the conventional equation of the spherical approximation, if the second term of the left-hand side is neglected. ${ }^{13)}$ This means that the second term of the left-hand side represents the effect of the nonspherical distribution of electrons, which has been disregarded in the conventional spherical approximation.

Multiplying $\frac{1}{r} p_{N_{1}}^{\sigma}(r)^{*} Y_{L_{1}}(\hat{\mathbf{r}})^{*}$ on both sides of eq. (3) and integrating over the whole space, we get

$$
\begin{aligned}
& \sum_{N} \sum_{L}\left\{\delta_{L_{1} L} O_{N_{1} N}^{\sigma}\left(\frac{\varepsilon_{N_{1} \sigma}^{0}+\varepsilon_{N \sigma}^{0}}{2}-\varepsilon_{i \sigma}\right)\right. \\
& \left.\quad+\int p_{N_{1}}^{\sigma}(r)^{*} V_{L_{1} L}^{\sigma}(r) p_{N}^{\sigma}(r) d r\right\} C_{N L}^{i \sigma}=0,
\end{aligned}
$$

where

$$
\begin{aligned}
O_{N_{1} N}^{\sigma}= & \int p_{N_{1}}^{\sigma}(r)^{*} p_{N}^{\sigma}(r) d r \\
V_{L_{1} L}^{\sigma}(r)= & \sum_{L^{\prime} \neq(00)} v_{L^{\prime}}^{\sigma}(r) \int Y_{L_{1}}(\hat{\mathbf{r}})^{*} Y_{L^{\prime}}(\hat{\mathbf{r}}) Y_{L}(\hat{\mathbf{r}}) d \hat{\mathbf{r}} \\
& +\sum_{L^{\prime} \neq(00)} v_{L^{\prime}}^{\sigma}(r)^{*} \int Y_{L_{1}}(\hat{\mathbf{r}})^{*} Y_{L^{\prime}}(\hat{\mathbf{r}})^{*} Y_{L}(\hat{\mathbf{r}}) d \hat{\mathbf{r}},
\end{aligned}
$$

and where $\varepsilon_{N \sigma}^{0}$ are the eigenvalues for $\hat{H}_{0}^{\sigma}$. The angular integrations in eq. (6) are rewritten by using the Wigner $3 \mathrm{j}$-symbols. According to the properties of the Wigner $3 \mathrm{j}$-symbols, matrix elements of eq. (6) are zero unless $l_{1}+l^{\prime}+l=$ even, $\quad\left|l_{1}-l\right| \leq l^{\prime} \leq l_{1}+l$ and $-m_{1}+m=$ $\pm m^{\prime}{ }^{13)}$ These conditions also determines the upper limit of the summation of eq. (1).

Equation (4) is just the generalized eigenvalue problem. If the matrix elements, $O_{N_{1} N}^{\sigma}$ and $V_{N_{1} N}^{\sigma}(r)$, and the energy spectra, $\varepsilon_{N \sigma}^{0}$, are given, then we can obtain the eigenvalues, $\varepsilon_{i \sigma}$, and eigenfunctions, $\left\{C_{N L}^{i \sigma}\right\}$. In what follows, we shall show the calculation procedure of the present scheme. We first give a starting potential in some usual way, for example via the LDA calculation within the spherical approximation (step 1). In order to prepare the radial basis functions $p_{n l}^{\sigma}(r)$, the spherical part of the potential is derived. Using this potential, atomic structure calculations are performed (step 2). Next, using the basis functions and the corresponding spectra, the generalized eigenvalue problem is solved (step 3 ). The resultant eigenfunctions provide new potentials by means of the expressions given in Appendix (step 4). Here we check whether the spherical and nonspherical parts of potentials are converged or not (step 5). If the convergence is not yet obtained, we return to step 2 with the spherical potential calculated from the new potential. The calculations are repeated until the potentials are converged within some accuracy. The feature of the present scheme is that the basis functions are updated for each iteration by imposing the self-consistency on the potentials.

\section{Results and Discussions}

We perform the actual calculation for the first-row atoms; boron, carbon, nitrogen, oxygen, fluorine and neon atoms. Before showing results, we shall state two points of calculation details. First, in the expansion (2) we use five functions having the following quantum numbers:

$$
(n l m)=(100),(200),(211),(210),(21-1) .
$$

Correspondingly, the upper limit of the potential given by eq. (1) is determined from the properties of the Wigner $3 \mathrm{j}$ symbols. The expansion of the potential consists of the following terms:

$$
\begin{aligned}
(\operatorname{lm})= & (00),(11),(10),(1-1),(22), \\
& (21),(20),(2-1),(2-2) .
\end{aligned}
$$

Second, various kinds of input potentials are arranged so as to cover the multiple self-consistent solutions. This is also a striking feature of the present scheme. This time, we prepare the orbitals of the spherical approximations, i.e. $1 \mathrm{~s}, 2 \mathrm{~s}$ and $2 p$ orbitals, and take the linear combination of them to construct the input potentials. The $1 \mathrm{~s}$ and $2 \mathrm{~s}$ orbitals are used as they stand, while $2 p$ orbitals are variously transformed as follows:

$$
\left(\begin{array}{c}
\tilde{\phi}_{1}^{\sigma} \\
\tilde{\phi}_{2}^{\sigma} \\
\tilde{\phi}_{3}^{\sigma}
\end{array}\right)=\left(\begin{array}{ccc}
\cos \theta & \sin \theta & 0 \\
\sin \theta & -\cos \theta & 0 \\
0 & 0 & 1
\end{array}\right)\left(\begin{array}{ccc}
\cos \phi & 0 & \sin \phi \\
0 & 1 & 0 \\
\sin \phi & 0 & -\cos \phi
\end{array}\right)\left(\begin{array}{c}
\phi_{211}^{\sigma} \\
\phi_{210}^{\sigma} \\
\phi_{21-1}^{\sigma}
\end{array}\right),
$$

where $\phi_{211}^{\sigma}, \phi_{210}^{\sigma}$ and $\phi_{21-1}^{\sigma}$ are $2 \mathrm{p}$ orbitals of the spherical approximation. The input potentials are constructed from the orbitals $\left\{\tilde{\phi}_{1}^{\sigma}, \tilde{\phi}_{2}^{\sigma}, \tilde{\phi}_{3}^{\sigma}\right\}$ and $1 \mathrm{~s}, 2 \mathrm{~s}$ ones. Changing the angles $\theta$ and $\phi$ by $15^{\circ}$, respectively, a total of 288 kinds of orbitals are taken so as to construct the starting potentials $((360 / 15)$. $(180 / 15)=288)$.

Let us show the calculation results for each atom. Figures 1, 2, 3, 4, 5 and 6 show the energy spectra of the 

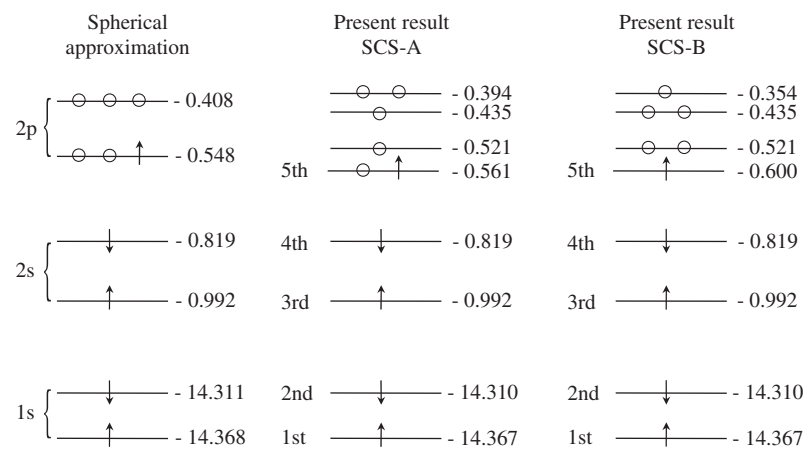

Fig. 1 Energy spectra for the neutral boron atom. The first column shows the results for the conventional spherical approximation. The second and third columns are the self-consistent solutions for the present scheme, which are denoted as "SCS-A" or "SCS-B". The up- and down-arrows denote the occupied states, and open circle the unoccupied states. All values are given in Rydberg Unit.
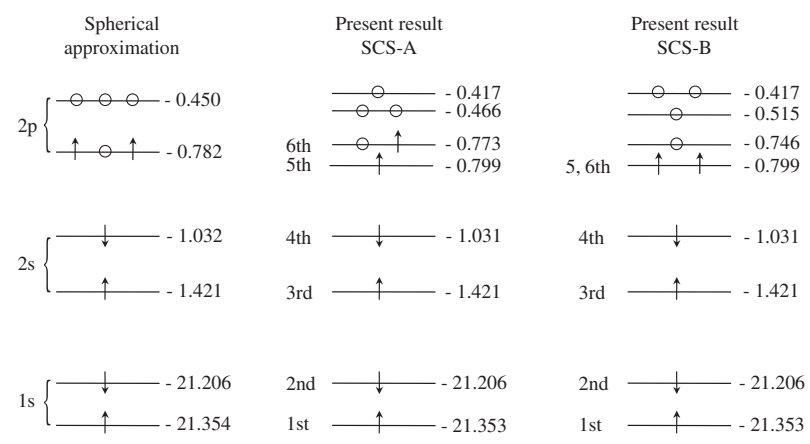

Fig. 2 Energy spectra for the neutral carbon atom.
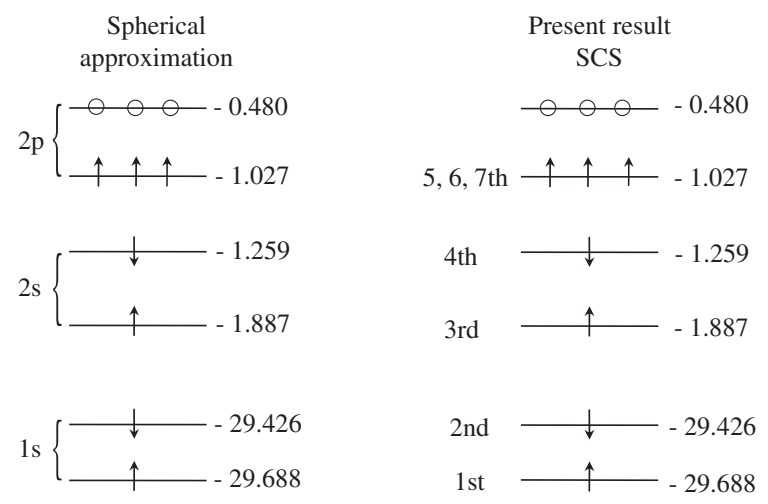

Fig. 3 Energy spectra for the neutral nitrogen atom.

present scheme, together with those of the conventional spherical approximation. For boron, carbon, oxygen and fluorine atoms (Figs. 1, 2, 4 and 5), the conventional 2p states are split into doubly degenerate levels and single one due to the effect of the nonspherical distribution of electrons. There exist two types of splitting, i.e., two types of the converged self-consistent solutions (SCS) can be found from the viewpoint of the splitting of energy levels. One is that the doubly degenerate levels are higher than the single one, and another is the opposite. They are denoted as "SCS-A" and "SCS-B", respectively, in Figs. 1, 2, 4 and 5. On the other hand, for nitrogen and neon atoms, there is no splitting in energy, as shown in Figs. 3 and 6. This means that the
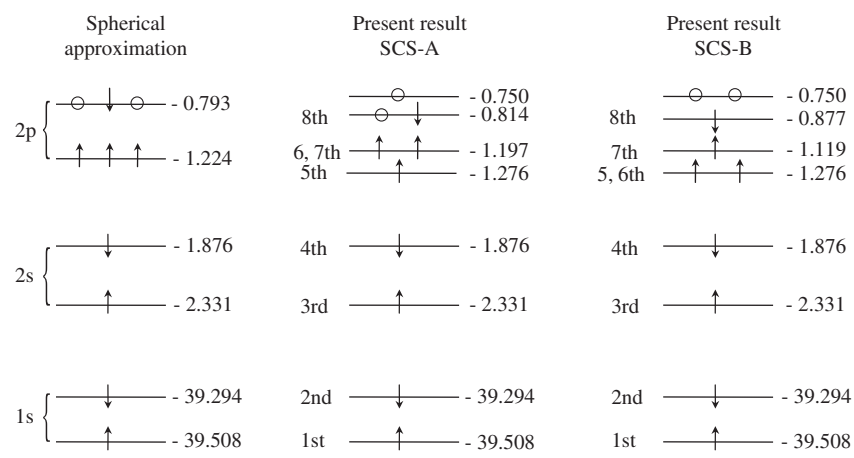

Fig. 4 Energy spectra for the neutral oxygen atom.
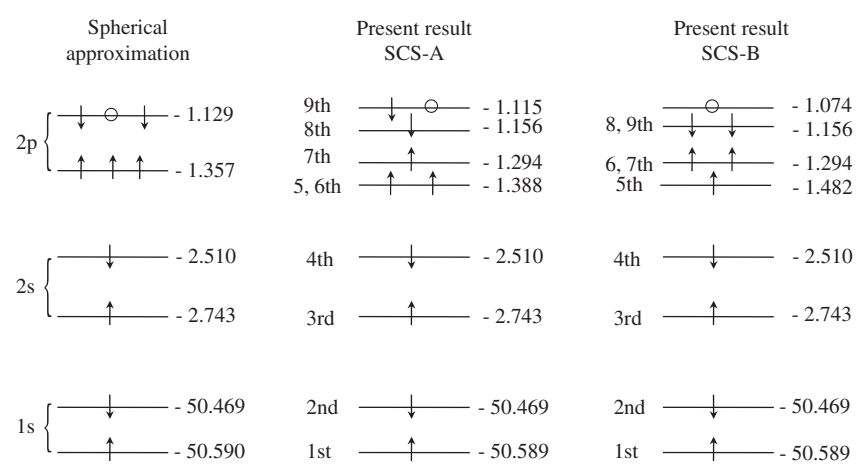

Fig. 5 Energy spectra for the neutral fluorine atom.

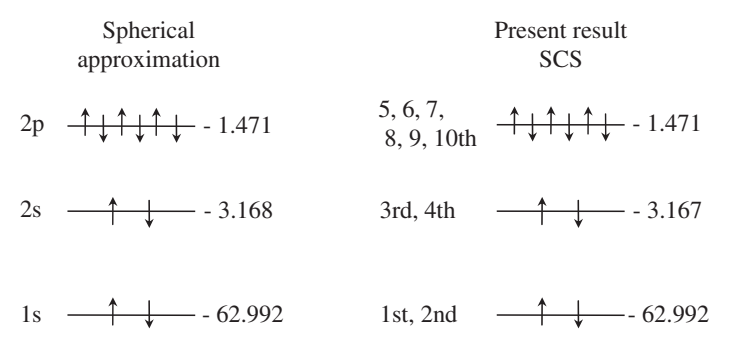

Fig. 6 Energy spectra for the neutral neon atom.

spherical approximation is reasonable for atoms having the outermost shell that is fully or half occupied since their electron densities are exactly spherical.

Here note that conventional $1 \mathrm{~s}$ and $2 \mathrm{~s}$ states are little influenced by the nonspherical distribution of electrons for all atoms. This is because the wave functions for $1 \mathrm{~s}$ and $2 \mathrm{~s}$ states are well localized near the nuclear where the spherical potential mainly caused by the nuclear is dominant.

In order to discuss the ground-state properties in more detail, we shall investigate the components of the eigenfunctions, i.e. the expansion coefficients of eq. (2). The expansion coefficients for the 1st, 2nd, 3rd and 4th states agree with those of the spherical approximation within the accuracy of $10^{-6}$ in all atoms. These results are consistent with the fact that energy levels for the 1st, 2nd, 3rd and 4th states are in a good agreement with the conventional $1 \mathrm{~s}$ and $2 \mathrm{~s}$ ones, respectively (Figs. 1, 2, 3, 4, 5 and 6). Concerning the expansion coefficients for nitrogen and neon atoms in which energy splittings are not observed, their results are also in a good agreement with the conventional ones. On the other hand, for atoms in which energy splittings are observed, the 
Table 1 The expansion coefficients of the 5th state for boron atom.

\begin{tabular}{lcccccc}
\hline & & \multicolumn{5}{c}{$(\mathrm{nlm})$} \\
& Energy [Ryd.] & $(100)$ & $(200)$ & $(21+1)$ & $(210)$ & $(21-1)$ \\
\hline SCS A-1 & -0.561 & 0.00000 & 0.00000 & 1.00000 & 0.00000 & 0.00000 \\
\hline SCS A-2 & -0.561 & 0.00000 & 0.00000 & 0.00000 & 0.00000 & 1.00000 \\
\hline SCS B-1 & -0.600 & 0.00000 & 0.00000 & 0.00000 & 1.00000 & 0.00000 \\
\hline SCS B-2 & -0.600 & 0.00000 & 0.00000 & $1 / \sqrt{2}$ & 0.00000 & $1 / \sqrt{2}$ \\
\hline SCS B-3 & -0.600 & 0.00000 & 0.00000 & $1 / \sqrt{2}$ & 0.00000 & $-1 / \sqrt{2}$ \\
\hline
\end{tabular}

Table 2 The expansion coefficients of the 5th and 6th states for carbon atom.

\begin{tabular}{ccccccc}
\hline & & \multicolumn{5}{c}{$(\mathrm{nlm})$} \\
& Energy [Ryd.] & $(100)$ & $(200)$ & $(21+1)$ & $(210)$ & $(21-1)$ \\
\hline \multirow{2}{*}{ SCS A-1 } & -0.799 & 0.00000 & 0.00000 & 0.00000 & 1.00000 & 0.00000 \\
& -0.773 & 0.00000 & 0.00000 & 1.00000 & 0.00000 & 0.00000 \\
\hline \multirow{2}{*}{ SCS A-2 } & -0.799 & 0.00000 & 0.00000 & 0.00000 & 1.00000 & 0.00000 \\
& -0.773 & 0.00000 & 0.00000 & 0.00000 & 0.00000 & 1.00000 \\
\hline \multirow{2}{*}{ SCS B-1 } & -0.799 & 0.00000 & 0.00000 & 1.00000 & 0.00000 & 0.00000 \\
& -0.799 & 0.00000 & 0.00000 & 0.00000 & 0.00000 & 1.00000 \\
\hline \multirow{2}{*}{ SCS B-2 } & -0.799 & 0.00000 & 0.00000 & 0.00000 & 1.00000 & 0.00000 \\
& -0.799 & 0.00000 & 0.00000 & $1 / \sqrt{2}$ & 0.00000 & $1 / \sqrt{2}$ \\
\hline \multirow{2}{*}{ SCS B-3 } & -0.799 & 0.00000 & 0.00000 & 0.00000 & 1.00000 & 0.00000 \\
& -0.799 & 0.00000 & 0.00000 & $1 / \sqrt{2}$ & 0.00000 & $-1 / \sqrt{2}$ \\
\hline
\end{tabular}

Table 3 The expansion coefficients of the 5th, 6th, 7th and 8th states for oxygen atom.

\begin{tabular}{ccccccc}
\hline & & & \multicolumn{5}{c}{$(\mathrm{nlm})$} \\
& Energy [Ryd.] & $(100)$ & $(200)$ & $(21+1)$ & $(210)$ & $(21-1)$ \\
\hline & -1.276 & 0.00000 & 0.00000 & 0.00000 & 1.00000 & 0.00000 \\
SCS A-1 & -1.197 & 0.00000 & 0.00000 & 1.00000 & 0.00000 & 0.00000 \\
& -1.197 & 0.00000 & 0.00000 & 0.00000 & 0.00000 & 1.00000 \\
& -0.814 & 0.00000 & 0.00000 & 1.00000 & 0.00000 & 0.00000 \\
\hline & -1.276 & 0.00000 & 0.00000 & 0.00000 & 1.00000 & 0.00000 \\
SCS A-2 & -1.197 & 0.00000 & 0.00000 & 1.00000 & 0.00000 & 0.00000 \\
& -1.197 & 0.00000 & 0.00000 & 0.00000 & 0.00000 & 1.00000 \\
& -0.814 & 0.00000 & 0.00000 & 0.00000 & 0.00000 & 1.00000 \\
\hline & -1.276 & 0.00000 & 0.00000 & 1.00000 & 0.00000 & 0.00000 \\
SCS B-1 & -1.276 & 0.00000 & 0.00000 & 0.00000 & 0.00000 & 1.00000 \\
& -1.119 & 0.00000 & 0.00000 & 0.00000 & 1.00000 & 0.00000 \\
& -0.877 & 0.00000 & 0.00000 & 0.00000 & 1.00000 & 0.00000 \\
\hline & -1.276 & 0.00000 & 0.00000 & 0.00000 & 1.00000 & 0.00000 \\
SCS B-2 & -1.276 & 0.00000 & 0.00000 & $1 / \sqrt{2}$ & 0.00000 & $-1 / \sqrt{2}$ \\
& -1.119 & 0.00000 & 0.00000 & $1 / \sqrt{2}$ & 0.00000 & $1 / \sqrt{2}$ \\
& -0.877 & 0.00000 & 0.00000 & $1 / \sqrt{2}$ & 0.00000 & $1 / \sqrt{2}$ \\
\hline & -1.276 & 0.00000 & 0.00000 & 0.00000 & 1.00000 & 0.00000 \\
SCS B-3 & -1.276 & 0.00000 & 0.00000 & $1 / \sqrt{2}$ & 0.00000 & $1 / \sqrt{2}$ \\
& -1.119 & 0.00000 & 0.00000 & $1 / \sqrt{2}$ & 0.00000 & $-1 / \sqrt{2}$ \\
& -0.877 & 0.00000 & 0.00000 & $1 / \sqrt{2}$ & 0.00000 & $-1 / \sqrt{2}$ \\
\hline
\end{tabular}

nonspherical distribution of electrons influences the outer orbitals directly. The expansion coefficients for these atoms are shown in Tables $1,2,3$ and 4 . In the conventional spherical approximation, electrons are distributed into each shell in an equal weight, ${ }^{25)}$ while in boron, carbon, oxygen
Table 4 The expansion coefficients of the 5th, 6th, 7th, 8th and 9th states for fluorine atom.

\begin{tabular}{ccccccc}
\hline & & & \multicolumn{5}{c}{$(\mathrm{nlm})$} \\
& Energy [Ryd.] & $(100)$ & $(200)$ & $(21+1)$ & $(210)$ & $(21-1)$ \\
\hline & -1.388 & 0.00000 & 0.00000 & 1.00000 & 0.00000 & 0.00000 \\
SCS A-1 & -1.388 & 0.00000 & 0.00000 & 0.00000 & 0.00000 & 1.00000 \\
& -1.294 & 0.00000 & 0.00000 & 0.00000 & 1.00000 & 0.00000 \\
& -1.156 & 0.00000 & 0.00000 & 0.00000 & 1.00000 & 0.00000 \\
& -1.115 & 0.00000 & 0.00000 & 1.00000 & 0.00000 & 0.00000 \\
\hline & -1.388 & 0.00000 & 0.00000 & 1.00000 & 0.00000 & 0.00000 \\
& -1.388 & 0.00000 & 0.00000 & 0.00000 & 0.00000 & 1.00000 \\
SCS A-2 & -1.294 & 0.00000 & 0.00000 & 0.00000 & 1.00000 & 0.00000 \\
& -1.156 & 0.00000 & 0.00000 & 0.00000 & 1.00000 & 0.00000 \\
& -1.115 & 0.00000 & 0.00000 & 0.00000 & 0.00000 & 1.00000 \\
\hline SCS B-1 & -1.482 & 0.00000 & 0.00000 & 0.00000 & 1.00000 & 0.00000 \\
& -1.294 & 0.00000 & 0.00000 & 1.00000 & 0.00000 & 0.00000 \\
& -1.294 & 0.00000 & 0.00000 & 0.00000 & 0.00000 & 1.00000 \\
& -1.156 & 0.00000 & 0.00000 & 1.00000 & 0.00000 & 0.00000 \\
& -1.156 & 0.00000 & 0.00000 & 0.00000 & 0.00000 & 1.00000 \\
\hline \multirow{5}{*}{ SCS B-3 } & -1.482 & 0.00000 & 0.00000 & $1 / \sqrt{2}$ & 0.00000 & $-1 / \sqrt{2}$ \\
& -1.294 & 0.00000 & 0.00000 & $1 / \sqrt{2}$ & 0.00000 & $1 / \sqrt{2}$ \\
& -1.294 & 0.00000 & 0.00000 & 0.00000 & 1.00000 & 0.00000 \\
& -1.156 & 0.00000 & 0.00000 & 0.00000 & 1.00000 & 0.00000 \\
& -1.294 & 0.00000 & 0.00000 & 0.00000 & 1.00000 & 0.00000 \\
& -1.156 & 0.00000 & 0.00000 & 0.00000 & 1.00000 & 0.00000 \\
& -1.156 & 0.00000 & 0.00000 & $1 / \sqrt{2}$ & 0.00000 & $1 / \sqrt{2}$ \\
\hline & -1.482 & 0.00000 & 0.00000 & $1 / \sqrt{2}$ & 0.00000 & $1 / \sqrt{2}$ \\
& -1.294 & 0.00000 & 0.00000 & $1 / \sqrt{2}$ & 0.0000 & $-1 / \sqrt{2}$ \\
& & & & & & \\
& & &
\end{tabular}

and fluorine atoms, components of each the converged SCS are partial to some of them. This partiality causes the polarization of the orbital angular momenta, which is socalled orbital polarization.

The orbital polarization is evaluated by calculating the expectation value of the operator of total orbital angular momentum with respect to the ground state. The ground state of the HFS approximation is given by a single Slater determinant that is written as

$$
\begin{aligned}
& \Psi\left(x_{1}, \cdots, x_{Z}\right) \\
& =\frac{1}{\sqrt{Z !}}\left|\begin{array}{ccccc}
\psi_{1 \alpha}\left(\mathbf{r}_{1}\right) \alpha\left(\eta_{1}\right) & \cdot & \cdot & \psi_{2 \beta}\left(\mathbf{r}_{1}\right) \beta\left(\eta_{1}\right) & \cdot \\
\cdot & \cdot & \cdot & \cdot \\
\cdot & & \cdot & \cdot & \cdot \\
\psi_{1 \alpha}\left(\mathbf{r}_{4}\right) \alpha\left(\eta_{4}\right) & \cdot & \cdot & \psi_{2 \beta}\left(\mathbf{r}_{4}\right) \beta\left(\eta_{4}\right) & \cdot \\
\cdot & \cdot & \cdot & \cdot
\end{array}\right|
\end{aligned}
$$

where $Z$ is the atomic number and $x_{i}$ denote the coordinates for $i$ th electron including spatial coordinate $\mathbf{r}_{i}$ and spin coordinate $\eta_{i}$, and where $\psi_{i \sigma}(\mathbf{r})$ is the solution of eq. (4), and where $\alpha(\eta)$ and $\beta(\eta)$ are wave functions for up- and downspins, respectively. Using eq. (8), the total orbital angular momentum, $L$, is calculated by

$$
\left\langle\Psi\left(x_{1}, \cdots x_{Z}\right)\left|\hat{\mathbf{L}}^{2}\right| \Psi\left(x_{1}, \cdots x_{Z}\right)\right\rangle=L(L+1),
$$

where $\hat{\mathbf{L}}=\sum_{i=1}^{Z} \hat{\mathbf{l}}_{i}$, and $\hat{\mathbf{l}}_{i}$ is the operator of the orbital angular momentum for the $i$ th electron. Similarly, the total spin angular momentum, $S$, is given by 
Table 5 The ground state values of the total orbital angular momentum and its z-component, $L$ and $L_{z}$, are shown in the 1 st and 2 nd columns, respectively. Also, the ground state values of the total spin angular momentum and its z-component, $S$ and $S_{z}$, are shown in the 3rd and 4th columns, respectively.

\begin{tabular}{|c|c|c|c|c|c|}
\hline $\begin{array}{c}\text { Atom } \\
\text { (atomic number) }\end{array}$ & state & $L$ & $L_{Z}$ & $S$ & $S_{Z}$ \\
\hline \multirow{3}{*}{ boron (5) } & SCS A-1 & 1.00000 & 1.00000 & 0.50000 & 0.50000 \\
\hline & SCS B-1, 2, 3 & 1.00000 & 0.00000 & 0.50000 & 0.50000 \\
\hline & SCS A-2 & 1.00000 & -1.00000 & 0.50000 & 0.50000 \\
\hline \multirow{3}{*}{ carbon (6) } & SCS A-1 & 1.00000 & 1.00000 & 1.00000 & 1.00000 \\
\hline & SCS B-1, 2, 3 & 1.00000 & 0.00000 & 1.00000 & 1.00000 \\
\hline & SCS A-2 & 1.00000 & -1.00000 & 1.00000 & 1.00000 \\
\hline nitrogen (7) & SCS & 0.00000 & 0.00000 & 1.50000 & 1.50000 \\
\hline \multirow{3}{*}{ oxygen (8) } & SCS A-1 & 1.00000 & 1.00000 & 1.01000 & 1.00000 \\
\hline & SCS B-1, 2, 3 & 1.00000 & 0.00000 & 1.01000 & 1.00000 \\
\hline & SCS A-2 & 1.00000 & -1.00000 & 1.01000 & 1.00000 \\
\hline \multirow{3}{*}{ fluorine (9) } & SCS A-1 & 1.00000 & 1.00000 & 0.52600 & 0.50000 \\
\hline & SCS B-1, 2, 3 & 1.00000 & 0.00000 & 0.52600 & 0.50000 \\
\hline & SCS A-2 & 1.00000 & -1.00000 & 0.52600 & 0.50000 \\
\hline neon (10) & SCS & 0.00000 & 0.00000 & 0.00000 & 0.00000 \\
\hline
\end{tabular}

$$
\left\langle\Psi\left(x_{1}, \cdots x_{Z}\right)\left|\hat{\mathbf{S}}^{2}\right| \Psi\left(x_{1}, \cdots x_{Z}\right)\right\rangle=S(S+1),
$$

where $\hat{\mathbf{S}}=\sum_{i=1}^{Z} \hat{\mathbf{s}}_{i}$, and $\hat{\mathbf{s}}_{i}$ is the operator of the spin angular momentum for the $i$ th electron. The results are shown in Table 5. Calculated values of $L$ and $S$ almost correspond to those of the multiplet term that is a ground state suggested by Hund's rule. In other words, the present scheme causes the orbital polarization for boron, carbon, oxygen and fluorine atoms both reasonably and definitely. Since the spherical approximation never brings it, we may say that the effect of the nonspherical distribution of electrons is one of the keys to the appearance of the orbital polarization.

At the end of this section, we would like to give a comment on the value of $L_{Z}$ and $S_{Z}$. The SCSs of the present scheme are classified into three kinds of states, which yield $L_{z}=1,0$ and -1 , respectively. Furthermore, the total energies of these states, which are also evaluated by taking the expectation values of the Hamiltonian with respect to eq. (8), coincide with each other. This is not surprising because the multiplet term with $L=1$ are essentially triply degenerate with respect to the orbital angular momentum, and its symptom seems to be seen in the ground states of the present scheme. Concerning the direction of spin, we fix it during the selfconsistent calculations. As a result, the value of $S_{Z}$ is fixed to be constant in the present results.

\section{Concluding Remarks}

In this paper, we present a scheme for calculating the atomic structures beyond the spherical approximation and investigate to what extent the single-particle picture of atomic systems needs to be modified for the first-row atoms. The results show that the energy levels for the outer orbitals of boron, carbon, oxygen and fluorine atoms are respectively split into two ones due to the effect of the nonspherical distribution of electrons. Two types of solutions can be obtained by setting various starting points of the self- consistent calculations, though only the SCS-B-type solution would be obtained in the previous works. ${ }^{7,8)}$ The magnitudes of energy splitting are not negligibly small for both types. These results would promote the modification of the singleparticle picture of the atomic systems.

Also, it is found that the orbital polarization emerges only by considering the nonspherical effect explicitly. Two types of solutions, i.e., SCS-A and SCS-B, correspond to the approximate states with $L_{z}= \pm 1$ and $L_{z}=0$, respectively. Since the atomic structures are undoubtedly fundamental for understanding the electronic structures of solids, the present results make ones reaffirm the fact that the effect of the nonspherical distribution of electrons may not be neglected also in solids. ${ }^{26-32)}$ It is also expected that the present scheme gives a good starting point to develop an alternative fullpotential method, besides the foundation of the hopping and Coulomb integrals appeared in the solid state calculations.

Although the present scheme shows the necessity of modifying the single-particle picture of atomic systems both conceptually and quantitatively, we have to consider the following effects that are neglected in the present calculations:

(1) enhancement of the expansion basis functions in eq. (2),

(2) treatment of the exchange energy beyond the Hartree-

Fock-Slater approximation.

Concerning the first effect, we here adopt only $1 \mathrm{~s}, 2 \mathrm{~s}$ and $2 \mathrm{p}$ orbitals in the expansion of the eigenfunctions. However we had better take more functions as the expansion basis functions so as to make the SCSs close to the actual ground states. Similarly, the second effect seems to be indispensable for describing the nonspherical effect in more rigorous way. After taking into account these neglecting points sufficiently, it will be possible to compare the total energy with that of quantum chemistry calculations, and determine whether it can be a standard of the cohesive energy of solids. But anyway, we can say within the knowledge obtained in this work that the effect of the nonspherical distribution of electrons cannot be neglected not only conceptually but also quantitatively in the study on the single-particle picture of atomic systems.

\section{Acknowledgements}

This work was partially supported by Grant-in-Aid for Scientific Research (No. 19540399) and for Scientific Research in Priority Areas (No. 17064006) of The Ministry of Education, Culture, Sports, Science and Technology, Japan.

\section{Appendix: Expressions for the Potentials}

In this appendix, we present the expressions for the spherical and nonspherical parts of the effective potential. The effective potential consists of three terms, i.e., the nuclear, Hartree and exchange potentials, which are given by

$V_{\sigma}(\mathbf{r})=-\frac{2 Z}{r}+2 \int \frac{\rho\left(\mathbf{r}^{\prime}\right)}{\left|\mathbf{r}-\mathbf{r}^{\prime}\right|} d \mathbf{r}^{\prime}-6\left(\frac{3}{4 \pi}\right)^{\frac{1}{3}} \rho_{\sigma}(\mathbf{r})^{\frac{1}{3}}$,

where the exchange potential is simplified with the aid of the Slater approximation, ${ }^{23)}$ and where $\rho(\mathbf{r})$ and $\rho_{\sigma}(\mathbf{r})$ denote the electron density and electron density with $\sigma$-spin, 
respectively. Using eq. (2), they are written as

$$
\begin{aligned}
\rho(\mathbf{r}) & =\sum_{i \sigma}^{\text {occ. }}\left|\psi_{i \sigma}(\mathbf{r})\right|^{2} \\
& =\sum_{i \sigma}^{\text {occ. }} \frac{1}{r^{2}}\left|\sum_{n l m} C_{n l m}^{i \sigma} p_{n l}^{\sigma}(r) Y_{l m}(\hat{\mathbf{r}})\right|^{2}, \\
\rho_{\sigma}(\mathbf{r}) & =\sum_{i(\sigma \text {-spin })}^{\text {occ. }}\left|\psi_{i \sigma}(\mathbf{r})\right|^{2} \\
& =\sum_{i(\sigma \text {-spin })}^{\text {occ. }} \frac{1}{r^{2}}\left|\sum_{n l m} C_{n l m}^{i \sigma} p_{n l}^{\sigma}(r) Y_{l m}(\hat{\mathbf{r}})\right|^{2},
\end{aligned}
$$

where the sum of eq. (A.3) is over only the occupied states with $\sigma$-spin. What we need are

(i) spherical part of eq. (A.1), i.e., $V_{\sigma}(r)=\frac{1}{\sqrt{4 \pi}}\left\{v_{00}^{\sigma}(r)+\right.$ $\left.v_{00}^{\sigma}(r)^{*}\right\}$, that appears in $\hat{H}_{0}^{\sigma}$, and

(ii) nonspherical components of eq. $(\mathrm{A} \cdot 1)$, i.e., $v_{k q}^{\sigma}(r)$, that appear in eq. (3) or (6).

The above (i) is indispensable for deriving the basis functions $p_{n l}^{\sigma}(r)$ and corresponding spectra $\varepsilon_{n l}^{\sigma 0}$, which are also used in eq. (4) or (5).
Now let us show the explicit forms of (i) and (ii) by considering each term of eq. (A-1). As for the first term, we have no problem because it is exactly spherical. Also, the second term can be easily separated into the spherical and nonspherical parts by means of the multipole expansion of the Coulomb potential. Concerning the third term, we have to use an approximation so as to derive one third power of $\rho_{\sigma}(\mathbf{r})$. Using the composition relation for the spherical harmonics, $\rho_{\sigma}(\mathbf{r})$ is formally separated into spherical and nonspherical parts as follows:

$$
\rho_{\sigma}(\mathbf{r})=\rho_{\sigma}^{\mathrm{S}}(r)+\rho_{\sigma}^{\mathrm{NS}}(\mathbf{r}),
$$

with

$$
\begin{aligned}
\rho_{\sigma}^{\mathrm{S}}(r)= & \sum_{i(\sigma \text {-spin })}^{\text {occ. }} \frac{1}{r^{2}} \sum_{n_{1} l_{1} m_{1}} \sum_{n_{2} l_{2} m_{2}} C_{n_{1} l_{1} m_{1}}^{i \sigma}{ }^{*} C_{n_{2} l_{2} m_{2}}^{i \sigma} \\
& \times p_{n_{1} l_{1}}^{\sigma}(r)^{*} p_{n_{2} l_{2}}^{\sigma}(r) \frac{(-1)^{m_{2}}}{8 \pi} \sqrt{\left(2 l_{1}+1\right)\left(2 l_{2}+1\right)} \\
& \times\left(\begin{array}{ccc}
l_{1} & l_{2} & 0 \\
0 & 0 & 0
\end{array}\right)\left(\begin{array}{ccc}
l_{1} & l_{2} & 0 \\
m_{1} & -m_{2} & 0
\end{array}\right)+C . C .,
\end{aligned}
$$

and

$$
\begin{aligned}
\rho_{\sigma}^{\mathrm{NS}}(\mathbf{r})= & \sum_{i(\sigma \text {-spin })}^{\text {occ. }}\left|\psi_{i \sigma}(\mathbf{r})\right|^{2} \\
= & \sum_{i(\sigma \text {-spin })}^{\text {occ. }} \frac{1}{r^{2}} \sum_{n_{1} l_{1} m_{1}} \sum_{n_{2} l_{2} m_{2}} C_{n_{1} l_{1} m_{1}}^{i \sigma} C_{n_{2} l_{2} m_{2}}^{i \sigma} \\
& \times p_{n_{1} l_{1}}^{\sigma}(r)^{*} p_{n_{2} l_{2}}^{\sigma}(r) \frac{(-1)^{m_{2}}}{2} \sum_{\substack{k=\left|l_{1}-l_{2}\right| \\
\neq 0=-k}}^{l_{1}+l_{2}} \underset{\neq 0}{k}\left\{\frac{\left(2 l_{1}+1\right)\left(2 l_{2}+1\right)(2 k+1)}{4 \pi}\right\}^{\frac{1}{2}} \\
& \times\left(\begin{array}{lll}
l_{1} & l_{2} & k \\
0 & 0 & 0
\end{array}\right)\left(\begin{array}{ccc}
l_{1} & l_{2} & k \\
m_{1} & -m_{2} & q
\end{array}\right) Y_{k q}(\hat{\mathbf{r}})+C . C .
\end{aligned}
$$

Since $\rho_{\sigma}^{N S}(\mathbf{r})$ is constructed from electrons of the unfilled outermost shell alone, it is quite smaller than the spherical part of the electron density. That is to say, $\rho_{\sigma}^{\mathrm{S}}(r) \gg \rho_{\sigma}^{\mathrm{NS}}(\mathbf{r})$. Using this fact, we can get an approximate form of the one third power of $\rho_{\sigma}(\mathbf{r})$ as follows

$$
\rho_{\sigma}(\mathbf{r})^{\frac{1}{3}} \approx \rho_{\sigma}^{\mathrm{S}}(r)^{\frac{1}{3}}+\frac{1}{3} \rho_{\sigma}^{\mathrm{S}}(r)^{-\frac{2}{3}} \rho_{\sigma}^{\mathrm{NS}}(\mathbf{r}),
$$

The first and second terms turn to the spherical and nonspherical parts of the exchange potential, respectively.

Using these relations, the resultant forms of (i) and (ii) are, respectively, given by

$$
\begin{aligned}
V_{\sigma}(r)= & -\frac{2 Z}{r}+\sum_{i \sigma}^{\text {occ. }} \sum_{n_{1} l_{1} m_{1}} \sum_{n_{2} l_{2} m_{2}} C_{n_{1} l_{1} m_{1}}^{i \sigma}{ }^{*} C_{n_{2} l_{2} m_{2}}^{i \sigma} \int d r^{\prime} \frac{1}{r_{>}} p_{n_{1} l_{1}}^{\sigma}\left(r^{\prime}\right)^{*} p_{n_{2} l_{2}}^{\sigma}\left(r^{\prime}\right) \\
& \times 2(-1)^{m_{1}} \sqrt{\left(2 l_{1}+1\right)\left(2 l_{2}+1\right)}\left(\begin{array}{ccc}
l_{1} & l_{2} & 0 \\
0 & 0 & 0
\end{array}\right)\left(\begin{array}{ccc}
l_{1} & l_{2} & 0 \\
-m_{1} & m_{2} & 0
\end{array}\right)-6\left(\frac{3}{4 \pi}\right)^{\frac{1}{3}} \rho_{\sigma}^{\mathrm{S}}(r)^{\frac{1}{3}}, \\
v_{k q}^{\sigma}(r)= & \sum_{i \sigma}^{\text {occ. }} \sum_{n_{1} l_{1} m_{1}} \sum_{n_{2} l_{2} m_{2}} C_{n_{1} l_{1} m_{1}}^{i \sigma} C_{n_{2} l_{2} m_{2}}^{i \sigma} \int d r^{\prime} \frac{r_{<}^{k}}{r_{>}^{k+1}} p_{n_{1} l_{1}}^{\sigma}\left(r^{\prime}\right)^{*} p_{n_{2} l_{2}}^{\sigma}\left(r^{\prime}\right) \\
& \times(-1)^{m_{2}} \sqrt{\frac{4 \pi\left(2 l_{1}+1\right)\left(2 l_{2}+1\right)}{2 k+1}}\left(\begin{array}{ccc}
l_{1} & l_{2} & k \\
0 & 0 & 0
\end{array}\right)\left(\begin{array}{ccc}
l_{1} & l_{2} & k \\
m_{1} & -m_{2} & q
\end{array}\right) \\
& -2\left(\frac{3}{4 \pi}\right)^{\frac{1}{3}} \rho_{\sigma}^{S}(r)^{-\frac{2}{3}} \sum_{i(\sigma-\text { spin })}^{\text {occ. }} \frac{1}{r^{2}} \sum_{n_{1} l_{1} m_{1}} \sum_{n_{2} l_{2} m_{2}} C_{n_{1} l_{1} m_{1}}^{* \sigma} C_{n_{2} l_{2} m_{2}}^{i \sigma} p_{n_{1} l_{1}}^{\sigma}(r)^{*} p_{n_{2} l_{2}}^{\sigma}(r) \\
& \times \frac{(-1)^{m_{2}}}{2}\left\{\frac{\left(2 l_{1}+1\right)\left(2 l_{2}+1\right)(2 k+1)}{4 \pi}\right\}^{\frac{1}{2}}\left(\begin{array}{ccc}
l_{1} & l_{2} & k \\
0 & 0 & 0
\end{array}\right)\left(\begin{array}{ccc}
l_{1} & l_{2} & k \\
m_{1} & -m_{2} & q
\end{array}\right) .
\end{aligned}
$$

Here $\left(\begin{array}{ccc}l_{1} & l_{2} & l_{3} \\ m_{1} & m_{2} & m_{3}\end{array}\right)$ is the Wigner $3 j$ symbol, ${ }^{13)}$ and $\rho_{\sigma}^{\mathrm{S}}(r)$ is given by eq. (A.5). 


\section{REFERENCES}

1) N. F. Mott: Proc. Roy. Soc. (London) A62 (1949) 416.

2) P. W. Anderson: Phys. Rev. 115 (1959) 2.

3) W. A. Harrison: Electronic Structure and the Properties of Solids-The Physics of the Chemical Bonds (W. H. Freeman and Co., San Francisco, 1980).

4) V. I. Anisimov, J. Zaanen and O. K. Andersen: Phys. Rev. B 44 (1991) 943.

5) A. I. Liechtenstein, V. I. Anisimov and J. Zaanen: Phys. Rev. B 52 (1995) R5468.

6) J. C. Slater: The Calculation of Molecular Orbitals (John Wiley \& Sons, New York, 1979).

7) J. F. Janak and A. R. Williams: Phys. Rev. B 23 (1981) 6301.

8) F. W. Kutzler and G. S. Painter: Phys. Rev. Lett. 59 (1987) 1285.

9) A. D. Becke: Int. J. Quantum Chem. 27 (1985) 585.

10) A. D. Becke: J. Chem. Phys. 117 (2002) 6935.

11) E. Orestes, T. Marcasso and K. Capelle: Phys. Rev. A 68 (2003) 022105 .

12) E. Orestes, A. B. F. da Silva and K. Capelle: Int. J. Quantum Chem. 103 (2005) 516.

13) A. Messiah: Quantum Mechanics (Dover Publications, New York, 1999).

14) P. Hohenberg and W. Kohn: Phys. Rev. 136 (1964) B864.
15) W. Kohn and L. J. Sham: Phys. Rev. 140 (1965) A1133.

16) G. Vignale and M. Rasolt: Phys. Rev. Lett. 59 (1987) 2360.

17) G. Vignale and M. Rasolt: Phys. Rev. B 37 (1988) 10685.

18) M. Higuchi and A. Hasegawa: J. Phys. Soc. Jpn. 66 (1997) 149.

19) M. Higuchi and A. Hasegawa: J. Phys. Soc. Jpn. 67 (1998) 2037.

20) D. R. Hartree: Proc. Cambridge Phil. Soc. 24 (1928) 111.

21) V. Fock: Z. Phys. 61 (1930) 126.

22) V. Fock: Z. Phys. 62 (1930) 795.

23) J. C. Slater: Phys. Rev. 81 (1951) 385.

24) F. Herman and S. Skillman: Atomic Structure Calculations (PrenticeHall Inc., New Jersey, 1963).

25) The spherical approximation exactly corresponds to the filling approximation of the outermost shell. For instance, see, S. E. Koonin: Computational Physics (Addison-Wesley, NY, 1986).

26) A. Narita and M. Higuchi: J. Phys. Soc. Jpn. 75 (2006) 024301.

27) O. Eriksson, B. Johansson, R. C. Albers, A. M. Boring and M. S. S. Brooks: Phys. Rev. B 42 (1990) 2707.

28) O. Eriksson, M. S. S. Brooks and B. Johansson: Phys. Rev. B 41 (1990) 7311.

29) M. S. S. Brooks, O. Eriksson, L. Severin and B. Johansson: Physica B 192 (1993) 39

30) T. Shishidou, T. Oguchi and T. Jo: Phys. Rev. B 59 (1999) 6813.

31) M. R. Norman: Phys. Rev. Lett. 64 (1990) 1162.

32) G. H. Daalderop, P. J. Kelly and M. F. H. Schuurmans: Phys. Rev. B 44 (1992) 12054. 